\title{
REPRESENTATIONS AND CLASSIFICATIONS OF STOCHASTIC PROCESSES
}

BY

\author{
DUDLEY PAUL JOHNSON
}

ABSTRACT. We show that to every stochastic process $X$ one can associate a unique collection $\left(\phi, \Phi_{+}, T(t), E(U), p^{*}\right)$ consisting of a linear space $\phi$, on which is defined a linear functional $p^{*}$, together with a convex subset $\Phi_{+}$which is invariant under the semigroup of operators $T(t)$ and the resolution of the identity $E(U)$. The joint distributions of $X$, there being one version for each $\phi \in \Phi_{+}$, are then given by

$$
P_{\phi}\left(X\left(t_{1}\right) \in U_{1}, \cdots, X\left(t_{1}+\cdots+t_{n}\right) \in U_{n}\right)=p^{*} E\left(U_{n}\right) T\left(t_{n}\right) \cdots E\left(U_{1}\right) T\left(t_{1}\right) \phi .
$$

To each $\phi$ contained in the extreme points $\Phi_{++}$of $\Phi_{+}$and each time $t$ we find a probability measure $P_{t}^{*}(\phi,$.$) on \Phi_{++}$such that $T(t) \phi=\int_{\Phi_{++}} \psi P_{t}^{*}(\phi, d \psi) . P_{t}^{*}$ is the transition probability function of a temporally homogeneous Markov process $X^{*}$ on $\phi_{++}$for which there exists a function $f$ such that $X=f\left(X^{*}\right)$. We show that in a certain sense $X^{*}$ is the smallest of all Markov processes $Y$ for which there exists a function $g$ with $X=g(Y)$. We then apply these results to a class of stochastic process in which future and past are independent given the present and the conditional distribution, on the past, of a collection of random variables in the future.

Introduction. It is the purpose of this paper to find minimal algebraic and Markov process representations of general stochastic processes and then to apply these representations to what we hope will turn out to be an interesting class of stochastic processes. The reader should note that our definition of a stochastic process will not be the standard one. Indeed we will define a stochastic process to be simply a convex set of probability measures on the sample path space which is closed under conditioning on the past. There are several reasons for our doing this. In the first place, a Markov process consists of a convex set of measures on the sample path space, one measure for each initial distribution, and these measures are closed under conditioning on the past. In the second place, suppose that an observer is looking at some random phenomenon which at some initial time is described by a particular measure on the sample path space. If a different

Received by the editors March 7, 1972 and, in revised form, March 3, 1973.

AMS (MOS) subject classifications (1970). Primary 60G05; Secondary 60J05, 60J25, $60 \mathrm{~K} 99$.

Key words and phrases. Stochastic process, algebraic representation, Markov process representation, Choquet's theorem, dual process, ¿ process. 
observer were to come along at a later random time, the phenomenon he observed would no longer be described by the original measure but by some convex combination of measures conditioned on the past. As a result we feel that it is best to think of the stochastic process as the collection of all possible measures which various observers might have to use to describe the phenomenon. Finally, we feel that there is some reason to believe that every random physical phenomenon is simply a temporally homogeneous Markor process in disguise. As a result, since the Markov process is a convex set of measures closed under conditioning on the past, we feel the original random phenomenon would also have this property.

Our first result is that to any stochastic process one can associate a unique algebraic structure whose main component consists of a semigroup of operators on a linear space. This association is well known in the case of temporally homogeneous Markov processes. The interested reader can refer to [6] to see how hitting time distributions for general stochastic processes can be found, using the algebraic representation, as the solution of an abstract Dirichlet problem.

Having defined a stochastic process and having constructed an algebraic representation for it, we then concern ourselves with the problem of representing the stochastic process as a function of a temporally homogeneous Markor process. We feel that this problem is an important one. In the first place, once having reduced a stochastic process to a function of a particular temporally homogeneous Markov process, we can then use the well-developed theory of Markov processes to study the original process. In the second place, we feel that in nature stochastic processes are Markovian and that the only reason we observe non-Markovian processes is that we are not observing enough; that there is some other variable which, taken together with ours, would make the process Markovian. For example, the number of cancerous cells in the body, as a function of time, is probably not Markovian. Yet one feels that there is probably some other observable, such as the amount of some substance in the body, which together with the number of cancerous cells would form a temporally homogeneous Markov process. This other observable would then be treated as the cause of the cancerous growth and its regulation would then hopefully regulate the growth of the cancer. We feel that finding a Markov process representation of a given stochastic process is equivalent to finding what is causing the process to behave as it does. Unfortunately one can find many Markov process representations of a given stochastic process. The problem is to find the simplest. In [5] we gave a method for constructing Markov process representations for general stochastic processes, but we did not show that the resulting representation was the best. In this paper we have divided stochastic processes into simple and nonsimple ones. We then order all Markov representations for a given stochastic process, and show first that for simple processes there is a unique minimal Markov process representation and that it is 
precisely the one constructed in [5]. We then show for discrete time stochastic processes, whether simple or not, that the minimal Markov process representations can be constructed using a variation of the construction used in [5]. Unfortunately, this representation need not be unique for nonsimple stochastic processes.

Finally we introduce classes of stochastic processes which we call $\Xi$ processes. These are generalizations of temporally homogeneous Markov processes and include the basic Bush-Mosteller [1] model for learning. Their defining property is that past and future are independent given the present and the conditional distribution, on the past, of a collection $\Xi$ of random variables in the future. We describe the algebraic representations of $\Xi$ processes and then construct explicitly the minimal Markov process representation for a particular example.

1. Definitions and statement of results. Let $\mathbb{N}=\mathbb{M}(\Omega, \mathcal{F})$ be the space of all finite valued signed measures on the measurable space $(\Omega, \mathfrak{F})$ of all functions $\omega$ mapping a subset $T$ of $[0, \infty)$ into a measurable space $(\mathcal{C}, \Sigma)$ where $\mathcal{F}$ is the $\sigma$-field generated by the events $X(t)(\omega)=\omega(t) \in U \in \Sigma$ and where $\mathcal{S}$ is a separable compact metric space with Borel sets $\Sigma$. Let $T(t), t \in T$, be the semigroup of linear operators on $\mathbb{N}$ defined by $T(t) \mu\left(X\left(t_{1}\right) \in U_{1}, \ldots, X\left(t_{n}\right) \in U_{n}\right)=$ $\mu\left(X\left(t+t_{1}\right) \in U_{1}, \cdots, X\left(t+t_{n}\right) \in U_{n}\right) ; E(U), U \in \Sigma$, be the resolution of the identity on $\pi$ defined by

$$
E(U) \mu(\Lambda)=\mu(X(0) \in U, \Lambda),
$$

and $p^{*}$ be the linear functional on $\pi$ defined by $p^{*} \mu=\mu(\Omega)$. We define a stochastic process to be any convex subset $\Phi_{+}$of probability measures in $\pi$ which has the property that the positive cone which it generates is invariant under $T(t), t \in T$, and $E(U), U \in \Sigma$, and closed in the weak* topology over the continuous functions in the product topology of $\Omega$. We denote by $\Phi$ the smallest linear space in $\pi$ containing $\Phi_{+}$and we define the algebraic representation of the stochastic process $\Phi_{+}$to be the collection $X=\left(\Phi, \Phi_{+}, T(t), E(U), p^{*}\right)$. Clearly if $\phi \in \Phi_{+}$, then we can calculate the joint distributions of $\phi$ from the algebraic representation $X$ using the easily verifiable fact that

$$
\phi\left(X\left(t_{1}\right) \in U_{1}, \cdots, X\left(t_{1}+\cdots+t_{n}\right) \in U_{n}\right)=p^{*} E\left(U_{n}\right) T\left(t_{n}\right) \cdots E\left(U_{1}\right) T\left(t_{1}\right) \phi .
$$

Two collections

$$
X=\left(\Phi, \Phi_{+}, T(t), E(U), p^{*}\right) \text { and } \hat{X}=\left(\hat{\Phi}, \hat{\Phi}_{+}, \hat{T}(t), \hat{E}(U), \hat{p}^{*}\right)
$$

will be called isomorphic if there exists a one-to-one linear mapping $\gamma$ of $\Phi$ onto $\hat{\Phi}$ such that

(i) $\gamma \Phi_{+}=\hat{\Phi}_{+}$

(ii) $\gamma T(t) \phi=\hat{T}(t) \gamma \phi$, 
(iii) $\gamma E(U) \phi=\hat{E}(U) \gamma \phi$,

(iv) $p^{*} \phi=\hat{p}^{*} \gamma \phi$.

With this in mind we have the following theorem.

Theorem I. A necessary and sufficient condition that a collection $X=$ $\left(\Phi, \Phi_{+}, T(t), E(U), p^{*}\right)$ be isomorphic to the algebraic representation of a stochas. tic process is that:

(1) $\Phi$ is a linear space generated by the convex subset $\Phi_{+}$.

(2) $T(t), t \in T$, is a semigroup of linear operators on $\Phi$ with $T(0)=l$.

(3) $E(U), U \in \Sigma$, is a family of linear operators on $\Phi$ wbicb is a resolution of the identity. That is to say,

$$
E(U \cup V)=E(U)+E(V) \text { if } U \cap V=\varnothing, E(U \cap V)=E(U) E(V) \text { and } E(\delta)=I \text {. }
$$

(4) $p^{*}$ is a linear functional on $\Phi$ for whicb $p^{*} T(t) \phi=p^{*} \phi$, $p^{*} E\left(U_{n}\right) T\left(t_{n}\right) \ldots E\left(U_{1}\right) T\left(t_{1}\right) \phi$ is continuous under monotone limits in $U_{1}, \ldots$, $U_{n}$ and $\phi=0$ whenever $p^{*} E\left(U_{n}\right) T\left(t_{n}\right) \ldots E\left(U_{1}\right) T\left(t_{1}\right) \phi=0$ for all $U_{1}, \ldots, U_{n}$ in $\Sigma$ and $t_{1}, \cdots, t_{n}$ in $T$.

(5) The positive cone $R_{+} \Phi_{+}$is invariant under the operators $T(t), t \in T$, and $E(U), U \in \Sigma$, and for eacb $\phi \in \Phi_{+}, p^{*} \phi=1$ and $p^{*} E\left(U_{n}\right) T\left(t_{n}\right) \ldots E\left(U_{1}\right) T\left(t_{1}\right) \phi \geq 0$. Furthermore $\Phi_{+}$is closed in the topology generated by the neighbourboods

$$
\begin{array}{r}
N\left(\epsilon, f_{1}, \cdots, f_{m}, t_{1}, \cdots, t_{n}\right)(\phi) \\
=\left\{\psi:\left|\int \cdots \int f_{i}\left(u_{1}, \cdots, u_{n}\right) p^{*} E\left(d u_{n}\right) T\left(t_{n}\right) \cdots E\left(d u_{1}\right) T\left(t_{1}\right)(\phi-\psi)\right|<\epsilon,\right. \\
i=1, \cdots, m\}
\end{array}
$$

where $f_{1}, \ldots, f_{m}$ are continuous functions on $\delta^{n}, t_{1}, \ldots, t_{n}$ are in $T$ and $\epsilon>0$.

For example, any temporally homogeneous strong Markov process will be a stochastic process under the above definition. Indeed, if $\mathcal{S}$ is the state space, then up to an isomorphism $\Phi$ would be the set of all measures on $\delta, \Phi_{+}$would be the set of all probability measures on $\delta, T(t)$ wuold be the semigroup of operators on $\Phi$ defined by

$$
(T(t) \phi)(U)=\int \phi(d u) P_{t}(u, U)
$$

where $P_{t}$ is the transition probability function, $E(U)$ would be the resolution of the identity on $\Phi$ defined by $(E(V) \phi)(U)=\phi(U \cap V)$ and $p^{*}$ would be the linear functional on $\Phi$ defined by $p^{*} \phi=\phi(\delta)$.

A temporally homogeneous Markor process $Y$ on a measurable state space $(\mathcal{J}, \mathfrak{B})$ will be called a perfect representation of the stochastic process $X$ if 
there exist measurable functions $f$ and $\theta$ mapping $\mathcal{T}$ into $\mathcal{S}$ and the set $\mathcal{P}(\mathcal{T})$ of all probability measures on $\mathscr{T}$ onto $\Phi_{+}$respectively such that, for all $m \in \mathcal{P}(\mathfrak{T})$,

$$
\theta m\left(X\left(t_{1}\right) \in U_{1}, \cdots, X\left(t_{n}\right) \in U_{n}\right)=P_{m}\left\{f \circ Y\left(t_{1}\right) \in U_{1}, \cdots, f \circ Y\left(t_{n}\right) \in U_{n}\right\}
$$

where $P_{m}$ is the distribution of $Y(t), t \in T$, when the initial distribution is $m$. In other words, the measures in $\Phi_{+}$can be interpreted as being precisely those measures derived from the temporally homogeneous Markov process $Y$ with various initial distributions. That is to say, any initial distribution of $Y$ yields via $f$ a measure in $\Phi_{+}$and any measure in $\Phi_{+}$derives from a particular initial distribution of $Y$. We will call a perfect representation measurable if there exists a measurable subset $\mathcal{T}^{\prime}$ of $\mathcal{T}$ such that $\theta$ restricted to $\mathcal{T}^{\prime}$ is a one-to-one mapping of $\mathcal{T}^{\prime}$ onto the set $\Phi_{++}$of extreme points of $\Phi_{+\cdot}$. The set $\boldsymbol{P}_{X}$ of all perfect. measurable representations of $X$ can be partially ordered by letting $Y^{\prime} \leq Y$ if:

(1) The state space $\left(\mathcal{J}^{\prime}, \mathfrak{B}^{\prime}\right)$ of $Y^{\prime}$ is contained in the state space $(\mathcal{T}, \mathfrak{B})$ of $Y$ in the sense that $\mathcal{T}^{\prime} \subset \mathcal{T}$ and $\mathfrak{B}^{\prime} \subset \mathfrak{B}, f^{\prime}$ is the restriction of $f$ to $Y^{\prime}$ and $\theta^{\prime}$ is the restriction of $\theta$ to $\mathscr{P}\left(\mathcal{J}^{\prime}\right)$.

(2) For each $b \in \mathcal{T} \backslash \mathcal{I}^{\prime}$ there exists a probability measure $M(b,$.$) on \mathcal{I}^{\prime}$ such that whenever $a \in \mathcal{I}^{\prime}$ and $U$ is a measurable set in $\mathcal{J}^{\prime}$, the transition probability functions $Q_{t}$ and $Q_{t}^{\prime}$ of $Y$ and $Y^{\prime}$ are related by

$$
Q_{t}^{\prime}(a, U)=Q_{t}(a, U)+\int_{\mathcal{J} \backslash g^{\prime}} Q_{t}(a, d b) M(b, U)
$$

$o r$, if the time parameter is discrete, by

$$
Q_{1}^{\prime}(a, U)=Q_{1}(a, U)+\int_{g \backslash g^{\prime}} Q_{1}(a, d b) M(b, U) .
$$

Intuitively, this means that $Y^{\prime}$ can be obtained from $Y$ by taking each state $b \in \mathfrak{T} \backslash \mathcal{T}^{\prime}$, splitting it into pieces and then assigning each piece to one of the states in $\mathcal{T}^{\prime}$, where the proportion of $b$ that is assigned to states in $U$ is $M(b, U)$.

If $X=\left(\Phi, \Phi_{+}, T(t), E(U), p^{*}\right)$ is a stochastic process with a countable time parameter $T$ and if $\Phi_{+}$is a simplex, then we shall say that $X$ is simple. When $X$ is simple it follows from Choquet's theorem (see [5], [7]) that for each $\phi$ in the set of extreme points $\Phi_{++}$of $\Phi_{+}$and each $t \in T$ there exist unique probability measures $P_{t}^{*}(\phi,$.$) on \Phi_{++}$for which

$$
T(t) \phi=\int_{\phi_{++}} \psi P_{t}^{*}(\phi, d \psi)
$$

Since the probability measures $P_{i}^{*}(\phi,$.$) are uniquely defined and since T(s+t)=$ $T(s) T(t)$, it follows that

$$
P_{s+t}^{*}(\phi, \cdot)=\int_{\phi_{++}} P_{s}^{*}(\phi, d \psi) P_{t}^{*}(\psi, \cdot)
$$


and so we construct a temporally homogeneous Markor process $X^{*}$ on $\Phi_{++}$with transition probability function $P_{t}^{*}$. If we let $f$ and $\theta$ be defined by the equations: $E(\{f \phi\}) \phi=\phi$ and $\theta m=\int_{\Phi_{++}} \phi m(d \phi)$, then it follows from [5] that for each $\phi \in \Phi_{+}$,

$$
\theta m\left\{X\left(t_{1}\right) \in U_{1}, \cdots, X\left(t_{n}\right) \in U_{n}\right\}=P_{m}\left\{f \circ Y\left(t_{1}\right) \in U_{1}, \cdots, f \circ Y\left(t_{n}\right) \in U_{n}\right\} .
$$

We shall call $X^{*}$ the dual of $X$. Clearly $X^{*}$ is a measurable perfect representation of $X$.

Theorem II. If the stocbastic process $X$ is simple, then $X^{*}$ is, up to an isomorpbism, the only minimal measurable perfect representation of $X$.

The reader should note that if $X$ is a temporally homogeneous Markov process, then $X$ is simple and $\Phi_{++}$is isomorphic to the set of all probability measures on $\delta$ whose support consists of a single point. In this case $\Phi_{++}$can be identified with $S$ and $P_{t}^{*}$, with this identification, is $P_{t}$. In other words, $X=X^{*}$.

If $X=\left(\Phi, \Phi_{+}, T(t), E(U), p^{*}\right)$ is a stochastic process which is not simple, then the above procedure might break down because $P_{t}, t \in T$, need not be a transition probability function. However, we can get around this difficulty when the time parameter is the nonnegative integers by defining the $n$-step transition probability function $P_{n}^{*}$ in terms of the one-step transition probability function $P^{*}$. Indeed, we know from Choquet's theorem that to each $\phi \in \Phi_{+}$there exists a probability measure $Q_{\phi}$ on $\Phi_{++}$such that $\phi=\int_{\Phi_{++}} \psi Q_{\phi}(d \psi)$. When $Q$ can be chosen so that for each measurable set $U \in \Phi_{++}, Q_{\phi}(U)$ is measurable in $\phi$, we will say that $X$ is measurable. It follows that when $X$ is measurable there exist probability measures $P^{*}(\phi,$.$) on \Phi_{++}$for which

$$
T \phi=\int_{\phi_{++}} \psi P^{*}(\phi, d \psi), \quad T=T(1),
$$

and for which $P^{*}(\cdot, U)$ is measurable for each measurable $U$ in $\Phi_{++\cdot}$ We define the dual $X^{*}$ of $X$ to be the temporally homogeneous Markov process on $\Phi_{++}$with one step transition probability function $P^{*}$. It should be noted that when $X$ is not simple, $X^{*}$ is not the same representation that was obtained in [5]. Taking $f$ and $\theta$ as before we have

Theorem III. If $X$ is a measurable discrete time stochastic process, then a necessary and sufficient condition that a measurable perfect representation of $X$ be minimal is that it be isomorphic to a version of $X^{*}$.

If $\Phi_{+}$consists of the set of all $\phi \in \Phi$ for which

$$
p^{*} E\left(U_{n}\right) T\left(t_{n}\right) \cdots E\left(U_{1}\right) T\left(t_{1}\right) \phi \geq 0, \quad p^{*} \phi=1,
$$

for all $U_{1}, \ldots, U_{n}$ in $\Sigma$ and $t_{1}, \ldots, t_{n}$ in $T$, then $X$ will be called an outer 
stochastic process and its algebraic representation will be written as $X=$ $\left(\Phi, T(t), E(U), p^{*}\right)$ since in that case $\Phi_{+}$is uniquely determined by $\Phi, T(t), E(U)$ and $p^{*}$. On the other hand, if there is a $\phi_{0} \in \Phi$ such that $\Phi_{+}$is generated by the closure of the convex hull of the points $E\left(U_{n}\right) T\left(t_{n}\right) \ldots E\left(U_{1}\right) T\left(t_{1}\right) \phi_{0}$, where $U_{1}, \ldots, U_{n}$ are in $\Sigma$ and $t_{1}, \ldots, t_{n}$ are in $T$, then $X$ will be called the inner stochastic process of $\phi_{0}$ and its algebraic representation will be written $X=$ $X\left(\phi_{0}\right)=\left(\Phi, T(t), E(U), p^{*}, \phi_{0}\right)$. To each inner stochastic process one can associate the outer stochastic process $\bar{X}=\left(\Phi, T(t), E(U), p^{*}\right)$. The reader should note that $\boldsymbol{\Phi}_{++}$is not in general equal to $\Phi_{++}$and $\bar{X}^{*}$ is not in general equal to $X^{*}$. An example of this phenomenon is given in $\$ 7$.

Suppose that $\Xi$ is a collection of bounded measurable real valued functions on $(\Omega, \mathcal{F})$ which includes the identity $1(\omega)=1$. An outer stochastic process $X=$ $\left(\Phi, T(t), E(U), p^{*}\right)$ will be called a $\Xi$ process if $\Phi_{+}$is the weak* closure of the set of all $\phi \in \Phi_{+}$for which, for any $t \in T$ and $\Lambda \in \mathcal{F}$,

$$
\phi\left(\Lambda_{t}^{+} \mid F_{t}\right)=\phi\left[\Lambda_{t}^{+} \mid X(t), E(\phi)\left(\xi_{t}^{+} \mid F_{t}\right), \xi \in \Xi\right] .
$$

Note that $\mathcal{F}_{t}$ is the $\sigma$ field generated by $X(u), u \leq t, \xi_{t}^{+}(\omega)=\xi\left(\omega_{t}^{+}\right)$where $\omega_{t}^{+}(u)=$ $\omega(t+u)$ and $\Lambda_{t}^{+}=\left[\omega: \omega_{t}^{+} \epsilon \Lambda\right]$. Also $E(\phi)$ denotes the expectation using the measure $\phi$.

Theorem IV. A necessary and sufficient condition that an outer stocbastic process $\hat{X}=\left(\hat{\Phi}, \hat{T}(t), \hat{E}(U), \hat{p}^{*}\right)$ be a $\Xi$ process is that $\hat{X}$ be isomorphic to a collection $X=\left(\Phi, T(t), E(U), p^{*}\right)$ where

(1) $\Phi$ is a linear subspace of the set of all functions mapping $\Sigma \times \Xi$ into $R$ wbicb are sucb that, for eacb $\xi \in \Xi, \phi(\cdot, \xi)$ is a measure.

(2) $E(V) \phi(U, \xi)=\phi(U V, \xi)$.

(3) $p^{*} \phi=\phi(\mathcal{S}, 1)$.

(4) $E(\hat{\phi})\left(\xi I_{U}\left(X_{0}\right)\right)=\phi(U, \xi)$.

Note that if $X=\left(\Phi, T(n), E(U), p^{*}\right)$ is a discrete time $\Xi$ process, then we can define the nonlinear operators $R(x), x \in \mathcal{S}$, via

$$
R(x) \phi(U, \xi)=E(\phi)\left(\xi_{1}^{+} I_{U}(X(1)) \mid X(0)=x\right)
$$

and it then follows that

$$
E(\phi)\left(\xi_{n+1}^{+} I U(X(n+1)) \mid X(1)=x_{1}, \cdots, X(n)=x_{n}\right)=R\left(x_{n}\right) \cdots R\left(x_{1}\right) \phi(U, \xi)
$$

and so

$$
\begin{aligned}
& \phi\left(X(0) \in U_{0}, \cdots, X(n) \in U_{n}\right) \\
& \quad=\int_{U_{0}} \phi\left(d x_{0}, 1\right) \int_{U_{1}} R\left(x_{0}\right) \phi\left(d x_{1}, 1\right) \cdots \int_{U_{n}} R\left(x_{n-1}\right) \cdots R\left(x_{0}\right) \phi\left(d x_{n}, 1\right) .
\end{aligned}
$$


In other words, $X$ is generated by first picking a point $x_{0}$ according to the distribution $\phi(\cdot, 1)$, then picking $x_{1}$ according to the distribution $R\left(x_{0}\right) \phi(\cdot, 1)$ and then $x_{2}$ according to the distribution $R\left(x_{1}\right) R\left(x_{0}\right) \phi(\cdot, 1)$ and so on. The reader can refer to [1], [3] and [4] for examples of stochastic processes similar to the $\boldsymbol{Z}$ processes defined here.

As an example, consider the discrete time $\Xi$ process where $\Xi=\Xi_{0}$ is the set of all functions $\xi$ of the form $\xi(\omega)=I_{U}(X(1)(\omega))$ where $I_{U}$ is the indicator of the Borel set $U$ in $R_{+}$. In this case $\delta=R_{+}$and $\Sigma$ is the Borel subset of $R_{+}$and so we can identify $\Phi$ with the linear space of functions $\Phi$ mapping $\Sigma \times \Sigma \rightarrow R$. We will assume that all of the functions $\phi \in \Phi$ are absolutely continuous in their second varaible with respect to the Lebesgue measure on $R_{+}$and we shall simply write the derivative of $\phi(U,$.$) with respect to the Lebesgue measure$ on $R_{+}$as $\phi^{\prime}(U, v)$. We will also assume that $\phi^{\prime}(U,.) \in C^{\infty}\left(R_{+}\right)$for each $U \in \Sigma$ and $\phi \in \Phi . \phi^{\prime}(u, v)$ will denote the density of $\phi$ with respect to the Lebesgue measure on $R_{+} \times R_{+}$when it exists. If $\phi(\{a\}, \delta)=1$ for some $a \in \mathcal{S}$, then $\phi^{\prime}(u, v)$ is to be $\phi^{\prime}(\delta, v) I_{\{a\}}(u)$. Finally, let $B$ be a bounded measurable function mapping $R_{+} \times R_{+} \times R_{+} \rightarrow R_{+}$which is a completely monotone function in its third variable and for which $B(x, y, y)=1$ for all $x$ and $y$ in $R_{+}$. It follows that there exist probability measures $a(x, y, \cdot)$ on $R_{+}$such that

$$
B(x, y, z)=\int_{0}^{\infty} e^{z t} \alpha(x, y, d t) .
$$

Theorem V. Let $X=\left(\Phi, T, E(U), p^{*}\right)$ be a $\Xi_{0}$ process with

$$
T \phi(U, V)=-\int_{V} d v \int_{U} d u \int_{0}^{\infty} \frac{\partial}{\partial v}\left[B(x, u, u+v) \phi^{\prime}(d x, u+v)\right]
$$

Tben:

$$
(R(x) \phi)^{\prime}(u, v)=-\frac{\partial}{\partial v}\left[B(x, u, u+v) \phi^{\prime}(x, u+v)\right] / \int_{0}^{\infty} \phi^{\prime}(x, z) d z .
$$

(2) The mapping

$$
\begin{aligned}
(a, b) \rightarrow \phi_{a, b}(U, V) & =\int_{V} e^{-b v} d v, & & a \in U, \\
& =0, & & a \notin U,
\end{aligned}
$$

is a bijection of $R_{+} \times R_{+}$onto $\Phi_{++}$and $f(a, b)=a$.

(3) $X^{*}$ bas transition probability function

$$
P(a, b: U, V)=\int_{U} d u \int_{(V-b)(1[0, \infty]} e^{-u(v+b)} \alpha(a, u, d v) .
$$

(4) A necessary and sufficient condition that $\phi$ be a version of $X$ (i.e. $\left.\phi \in \Phi_{+}\right)$is that for any $0 \leq x \leq y$, 


$$
\begin{gathered}
\phi(X(n+1)<x, X(0), \cdots, X(n), X(n+1)<y<X(n+1)+X(n+2)) \\
=\int_{0}^{x} B(X(n), \xi, y) d \xi / \int_{0}^{y} B(X(n), \xi, y) d \xi .
\end{gathered}
$$

2. Proof of Theorem I. The necessity of conditions (1) through ( 5 ) is clear. To prove the sufficiency, suppose that $X=\left(\Phi, \Phi_{+}, T(t), E(U), p^{*}\right)$ satisfies conditions (1) through (5). To each $\phi \in \Phi_{+}$for which $p^{*} \phi=1$ we get, via conditions (1) through (5) and the Kolmogorov extension theorem, a probability measure $\gamma \phi \in \mathbb{M}$ where

$$
\gamma \phi\left(X\left(t_{1}\right) \in U_{1}, \cdots, X\left(t_{1}+\cdots+t_{n}\right) \in U_{n}\right)=p^{*} E\left(U_{n}\right) T\left(t_{n}\right) \cdots E\left(U_{1}\right) T\left(t_{1}\right) \phi .
$$

Clearly $\hat{X}=\left(\hat{\Phi}, \hat{\Phi}_{+}, \hat{T}(t), \hat{E}(U), \hat{p}^{*}\right)$, where $\hat{\Phi}=\gamma \Phi, \hat{T}(t) \gamma \phi=\gamma T(t) \phi, \hat{E}(U) \gamma \phi=$ $\gamma E(U) \phi$ and $\hat{p}^{*} \gamma \phi=p^{*} \phi$ is a stochastic process. Furthermore, $\gamma$ is an isomorphism of $X$ onto $\hat{X}$. For example, $\gamma$ is oneto-one since $\gamma \phi=\gamma \psi$ implies that $p^{*} E\left(U_{n}\right) T\left(t_{n}\right) \ldots E\left(U_{1}\right) T\left(t_{1}\right)(\phi-\psi)=0$ for all choices of $U_{1}, \ldots, U_{n}$ in $\Sigma$ and $t_{1}, \ldots, t_{n}$ in $T$ and so, by condition (4) of Theorem $1, \phi=\psi$.

3. Proof of Theorem II. We first show that $\leq$ is indeed a partial ordering of $P_{X}$. Suppose that $Y \leq Y^{\prime} \leq Y^{\prime \prime}$. Then $\mathcal{T} \subseteq \mathcal{T}^{\prime} \subseteq \mathcal{T}^{\prime \prime}$ and there exist measures $M(b,$.$) for each b \in \mathcal{J}^{\prime} \backslash \mathcal{T}$ and $M^{\prime}(b,$.$) for each b \in \mathcal{T}^{\prime \prime} \backslash \mathcal{J}^{\prime}$ for which

$$
Q_{t}(a, U)=Q_{t}^{\prime}(a, U)+\int_{\mathcal{I}^{\prime} \backslash g} Q_{t}^{\prime}(a, d b) M(b, U)
$$

and

$$
Q_{t}^{\prime}(a, U)=Q_{t}^{\prime \prime}(a, U)+\int_{\mathcal{J}^{\prime \prime} \backslash \mathcal{J}^{\prime}} Q_{t}^{\prime \prime}(a, d b) M^{\prime}(b, U) \text {. }
$$

Setting $M(b, \cdot)=0$ for $b \in \mathcal{T}^{\prime} \backslash \mathcal{T}$ and $M^{\prime}(b, \cdot)=0$ for $b \in \mathcal{I}^{\prime \prime} \backslash \mathcal{J}^{\prime}$ gives us

$$
\begin{aligned}
& Q_{t}(a, U)=Q_{t}^{\prime \prime}(a, U)+\int_{\mathcal{g}^{\prime \prime} \backslash g^{\prime}} Q_{t}^{\prime \prime}(a, d b) M^{\prime}(b, U) \\
& +\int_{\mathcal{J}^{\prime} \backslash \mathcal{g}} Q_{t}^{\prime \prime}(a, d b) M(b, U)+\int_{\mathcal{I}^{\prime \prime} \backslash \mathcal{I}^{\prime}} Q_{t}^{\prime \prime}(a, d b) \int_{\mathcal{J}^{\prime} \backslash \mathcal{I}^{\prime}} M^{\prime}(b, d c) M(c, U) \\
& =Q_{t}^{\prime \prime}(a, U)+\int_{\mathcal{I}^{\prime \prime} \backslash \mathcal{J}} Q_{t}^{\prime \prime}(a, d b) M^{\prime \prime}(b, U)
\end{aligned}
$$

where

$$
M^{\prime \prime}(b, U)=M^{\prime}(b, U)+M(b, U)+\int_{g^{\prime} \backslash \mathcal{J}} M^{\prime}(b, d c) M(c, U) .
$$

Thus $Y \leq Y^{\prime} \leq Y^{\prime \prime}$ implies that $Y \leq Y^{\prime \prime}$. Since $\leq$ is reflexive and antisymmetric, it follows that $\left(\mathcal{P}_{X}, \leq\right)$ is a partially ordered system. Suppose that $Y$ is a measurable perfect representation of $X$. Then $Y$ is a temporally homogeneous Markov process on some state space $(\mathcal{T}, \Re)$ with transition probability function $P_{t}$, and 
there are functions $f$ and $\theta$ mapping $\mathscr{T}$ into $\mathcal{S}$ and $\mathscr{P}(\mathcal{T})$ onto $\Phi_{+}$respectively such that, for each $m \in \mathscr{P}(\mathfrak{T})$,

$$
\theta m\left\{X\left(t_{1}\right) \in U_{1}, \cdots, X\left(t_{n}\right) \in U_{n}\right\}=P_{m}\left\{f \circ Y\left(t_{1}\right) \in U_{1}, \cdots, f \circ Y\left(t_{n}\right) \in U_{n}\right\} .
$$

Since $Y$ is a measurable representation of $X$, we know that there exists a measurable subset $\mathcal{J}^{\prime}$ of $\mathfrak{T}$ consisting of exactly one point from each of the sets $\theta^{-1} \phi, \phi \in \Phi_{++}$For each $c \in \mathcal{T}$, let $m_{c}$ be the probability measure on $(\mathfrak{T}, \mathfrak{B})$ whose support is $\{c\}$. We know from Choquet's theorem, together with the fact that $X$ is simple, that there exists for each $c \in \mathfrak{I} \backslash \mathcal{I}^{\prime}$ a unique probability measure $Q_{c}(\cdot)$ on $\mathcal{J}^{\prime}$ for which

$$
\phi_{c}=\int_{g} \phi_{b} Q_{c}(d b), \quad \phi_{c}=\theta m_{c} .
$$

Let $P_{t}^{\prime}$ be defined by

$$
P_{t}^{\prime}(a, U)=P_{t}(a, U)+\int_{\mathcal{J} \backslash g^{\prime}} P_{t}(a, d b) Q_{b}(U) .
$$

Then for any $a \in \mathcal{T}^{\prime}$, we have

$$
\begin{aligned}
p^{*} E\left(U_{n}\right) T\left(t_{n}\right) \cdots E\left(U_{1}\right) T\left(t_{1}\right) \int_{g} \phi_{b} P_{t}(a, d b) \\
=\int_{g} p^{*} E\left(U_{n}\right) T\left(t_{n}\right) \cdots E\left(U_{1}\right) T\left(t_{1}\right) \phi_{b} P_{t}(a, d b) \\
=\int_{g} \phi_{b}\left(X\left(t_{1}\right) \in U_{1}, \cdots, X\left(t_{1}+\cdots+t_{n}\right) \in U_{n}\right) P_{t}(a, d b) \\
=\int_{g} P_{m_{b}}\left(f \circ Y\left(t_{1}\right) \in U_{1}, \cdots, f \circ Y\left(t_{1}+\cdots+t_{n}\right) \in U_{n}\right) P_{t}(a, d b) \\
=P_{m_{a}}\left(f \circ Y\left(t+t_{1}\right) \in U_{1}, \cdots, f \circ Y\left(t+t_{1}+\cdots+t_{n}\right) \in U_{n}\right) \\
=\phi_{a}\left(X\left(t+t_{1}\right) \in U_{1}, \cdots, X\left(t+t_{1}+\cdots+t_{n}\right) \in U_{n}\right) \\
=p^{*} E\left(U_{n}\right) T\left(t_{n}\right) \cdots E\left(U_{1}\right) T\left(t_{1}\right) T(t) \phi_{a} \\
=p^{*} E\left(U_{n}\right) T\left(t_{n}\right) \cdots E\left(U_{1}\right) T\left(t_{1}\right) \int_{\phi_{t+}} \psi P_{b}^{*}\left(\phi_{a}, d \psi\right) .
\end{aligned}
$$

Thus $\int_{\mathcal{g}} \phi_{b} P_{t}(a, d b)=\int_{\phi_{++}} \psi P_{t}^{*}\left(\phi_{a}, d \psi\right)$ and so

$$
\begin{aligned}
& \int_{g^{\prime}} \phi_{b} P_{t}^{\prime}(a, d b)=\int_{g^{\prime}} \phi_{b} P_{t}(a, d b)+\int_{g^{\prime}} \phi_{b}\left[\int_{g} \backslash g^{\prime} P_{t}(a, d c) Q_{c}(d b)\right] \\
& =\int_{g^{\prime}} \phi_{b} P_{t}(a, d b)+\int_{g \backslash g^{\prime}}\left[\int_{g} \phi_{b} Q_{c}(d b)\right] P_{t}(a, d c) \\
& =\int_{g^{\prime}} \phi_{b} P_{t}(a, d b)+\int_{\mathcal{g} \backslash g^{\prime}} \phi_{c} P_{t}(a, d c) \\
& =\int_{g} \phi_{b} P_{t}(a, d b)=\int_{\phi_{++}} \psi P_{t}^{*}\left(\phi_{a}, d \psi\right) \text {. }
\end{aligned}
$$


Since $\Phi_{+}$is a simplex, it follows that $P_{t}^{\prime}(a, d b)=P_{t}^{*}\left(\phi_{a}, d \phi_{b}\right)$ and so $X^{*}=Y^{\prime}$ $\leq Y$. Since $X^{*}$ is a perfect representation of $X$, see $[5]$, it follows that $X^{*}$ is the only minimal perfect representation of $X$ and the theorem is proved.

4. Proof of Theorem III. Since, for each $m \in \mathcal{P}\left(\Phi_{++}\right)$,

$$
\begin{aligned}
& (\theta m)\left(X(0) \in U_{0}, \cdots, X(n) \in U_{n}\right)=p^{*} E\left(U_{n}\right) T \cdots E\left(U_{1}\right) T E\left(U_{0}\right)(\theta m) \\
& =p^{*} E\left(U_{n}\right) T \ldots E\left(U_{1}\right) T E\left(U_{0}\right) \int_{\psi_{++}} \psi_{0} m\left(d \psi_{0}\right) \\
& =p^{*} E\left(U_{n}\right) T \cdots E\left(U_{1}\right) T \int_{f-1} \psi_{0} m\left(d \psi_{0}\right) \\
& =\int_{f-1} m\left(d \psi_{0}\right) p^{*} E\left(U_{n}\right) T \cdots E\left(U_{1}\right) T \psi_{0} \\
& =\int_{f^{-1} U_{0}} m\left(d \psi_{0}\right) p^{*} E\left(U_{n}\right) T \cdots E\left(U_{1}\right) \int_{\varphi_{++}} \psi_{1} P^{*}\left(\psi_{0}, d \psi_{1}\right) \\
& =\int_{f^{-1} U_{0}} m\left(d \psi_{0}\right) p^{*} E\left(U_{n}\right) T \cdots E\left(U_{2}\right) T \int_{T^{-1} U_{1}} \psi_{1} P^{*}\left(\psi_{0}, d \psi_{1}\right) \\
& =\int_{T^{-1} U_{0}} m\left(d \psi_{0}\right) \int_{f^{-1} U_{1}} P^{*}\left(\psi_{0}, d \psi_{1}\right) p^{*} E\left(U_{n}\right) T \ldots E\left(U_{2}\right) T \psi_{1} \\
& =\int_{f^{-1} U_{0}} m\left(d \psi_{0}\right) \int_{\Gamma^{-1} U_{1}} P^{*}\left(\psi_{0}, d \psi_{1}\right) \cdots \int_{f^{-1} U_{n}} P^{*}\left(\psi_{n-1}, d \psi_{n}\right) \\
& =P_{m}\left(f \circ X^{*}(0) \in U_{0}, \cdots, f \circ X^{*}(n) \in U_{n}\right)
\end{aligned}
$$

it follows that $X^{*}$ is a perfect representation of $X$. Suppose that $X^{*}$ is not minimal. Then there exists a subset $\Phi_{++}^{\prime}$ of $\Phi_{++}$and a probability measure $M\left(\phi,{ }^{\circ}\right)$ for each $\phi \in \Phi_{++} \backslash \Phi_{++}^{\prime}$ such that the temporally homogeneous Markov process $X^{\prime}$ on $\Phi_{++}^{\prime}$ with one step transition probability function

$$
P^{\prime}(\phi, \cdot)=P^{*}(\phi, \cdot)+\int_{\phi_{++} \backslash \varphi_{++}^{\prime}} P^{*}(\phi, d \psi) M(\psi, \cdot)
$$

is a perfect representation of $X$. That is to say,

$$
\theta m\left(X\left(t_{1}\right) \in U_{1}, \cdots, X\left(t_{n}\right) \in U_{n}\right)=P_{m}\left(f^{\prime} \circ Y\left(t_{1}\right) \in U_{1}, \ldots, f^{\prime} \circ Y\left(t_{n}\right) \in U_{n}\right)
$$

where $f^{\prime}$ and $\theta^{\prime}$ are the restrictions of $f$ and $\theta$ to $\Phi_{++}^{\prime}$ and $\mathscr{P}\left(\Phi_{++}^{\prime}\right)$ respectively. Let $\phi \in \Phi_{++} \backslash \Phi_{++\cdot}^{\prime}$ Then

$$
\begin{aligned}
& p^{*} E\left(U_{n}\right) T \cdots E\left(U_{1}\right) T E\left(U_{0}\right)(\theta m) \\
& =\int_{f^{\prime-1} U_{0}} m\left(d \psi_{0}\right) \int_{f^{\prime-1} U_{1}} P^{\prime}\left(\psi_{0}, d \psi\right) \cdots \int_{f^{\prime-1} U_{n}} P^{\prime}\left(\psi_{n-1}, d \psi_{n}\right) \\
& =\int_{, 1-1} m\left(d \psi_{0}\right) \psi_{0}\left(X(0) \in U_{0}, \ldots, X(n) \in U_{n}\right) \\
& =\int_{f^{-1} U_{0}} m\left(d \psi_{0}\right) p^{*} E\left(U_{n}\right) T \ldots E\left(U_{1}\right) T E\left(U_{0}\right) \psi_{0} \\
& =p^{*} E\left(U_{n}\right) T \cdots E\left(U_{1}\right) T E\left(U_{0}\right) \int_{f^{\prime-1} U_{0}} \psi_{0} m\left(d \psi_{0}\right) .
\end{aligned}
$$


Since this holds for all choices of $n, U_{0}, \ldots, U_{n}$, it follows that

$$
\phi=\int_{f^{1-1} U_{0}} \psi m(d \psi) \text {. }
$$

But $\phi \in \Phi_{++}$and $\phi \notin f^{\prime-1} U_{0} \subset \Phi_{++} \backslash \Phi_{++}^{\prime}$ and so we have a contradiction. Thus $X^{*}$ is minimal.

On the other hand, suppose that $Y$ is a measurable representation of $X$. Then there exists a measurable subset $\mathfrak{T}^{\prime}$ of $\mathfrak{T}$ consisting of exactly one point from each of the sets $\theta^{-1} \phi, \phi \in \Phi_{++}$. For each $c \in \mathcal{T}$, let $m_{c}$ be the probability measure on $(\mathcal{T}, \mathfrak{B})$ whose support is $\{c\}$. Since $X$ is measurable we know that for each $c \in \mathcal{I} \backslash \mathcal{J}^{\prime}$ we can find a probability measure $Q_{c}(\cdot)$ on $\mathcal{I}^{\prime}$ which is measurable in $c$ and which has the property that

$$
\phi_{c}=\int_{g} \phi_{b} Q_{c}(d b), \quad \phi_{c}=\theta m_{c} .
$$

Let $P^{\prime}$ be the one step transition probability function

$$
P^{\prime}(a, U)=P(a, U)+\int_{g \backslash g^{\prime}} P(a, d b) Q_{b}(U) .
$$

Then for any $a \in \mathcal{I}^{\prime}$ we have

$$
\begin{aligned}
p^{*} E & \left(U_{n}\right) T \cdots E\left(U_{1}\right) T E\left(U_{0}\right) \int_{g} \phi_{b} P(a, d b) \\
& =\int_{g} p^{*} E\left(U_{n}\right) T \cdots E\left(U_{1}\right) T E\left(U_{0}\right) \phi_{b} P(a, d b) \\
& =\int_{g} \phi_{b}\left(X(0) \in U_{0}, \ldots, X(n) \in U_{n}\right) P(a, d b) \\
& =\int_{g} P_{m_{b}}\left(f \circ Y(0) \in U_{0}, \cdots, f \circ Y(n) \in U_{n}\right) P(a, d b) \\
& =P_{m_{a}}\left(f \circ Y(1) \in U_{0}, \ldots, f \circ Y(n+1) \in U_{n}\right) \\
& =\phi_{a}\left(X(1) \in U_{0}, \cdots, X(n+1) \in U_{n}\right)=p^{*} E\left(U_{n}\right) T \cdots E\left(U_{0}\right) T \phi_{a} .
\end{aligned}
$$

Since our algebraic representation is reduced we can conclude that $T \phi_{a}=$ $\int_{g} \phi_{b} P(a, d b)$. Hence

$$
\begin{aligned}
\int_{g^{\prime}} \phi_{b} P^{\prime}(a, d b) & =\int_{g^{\prime}} \phi_{b} P(a, d b) \int_{g^{\prime}} \phi_{b}\left[\int_{\mathcal{g} \backslash g^{\prime}} P(a, d c) Q_{c}(d b)\right] \\
& =\int_{g^{\prime}} \phi_{b} P(a, d b)+\int_{\mathcal{g} \backslash g^{\prime}}\left[\int_{g^{\prime}} \phi_{b} Q_{c}(d b)\right] P(a, d c) \\
& =\int_{g^{\prime}} \phi_{b} P(a, d b)+\int_{\mathcal{J} \backslash g^{\prime}} \phi_{c} P(a, d c) \\
& =\int_{g} \phi_{b} P(a, d b)=T \phi_{a^{\circ}}
\end{aligned}
$$

Since $\mathcal{I}^{\prime} \subset \Phi_{++}$we conclude that $Y^{\prime}$ is a version of $X^{*}$ and the proof is completed. 
5. Proof of Theorem IV. To prove the sufficiency, suppose that the stochastic process $X=\left(\Phi, T(t), E(U), p^{*}\right)$ satisfies the conditions (1) through (4). To show that $X$ is a $\Xi$ process, we must show for all $\Lambda \in \mathcal{F}, \phi \in \Phi_{+}$and $t \in T$ that

$$
\phi\left(\Lambda_{t}^{+} \mid \mathcal{F}_{t}\right)=\phi\left[\Lambda_{t}^{+} \mid X(t), E(\phi)\left(\xi_{t}^{+} \mid \mathcal{F}_{t}\right), \xi \in \Xi\right] \text {. }
$$

But for any choice of $U_{1}, \ldots, U_{m+n}$ in $\Sigma$ and $s_{1}, \ldots, s_{m+n}$ in $T$, letting $\psi(k)=E\left(U_{k}\right) T\left(s_{k}\right) \ldots E\left(U_{1}\right) T\left(s_{1}\right) \phi$, we have

$$
\begin{aligned}
\phi\left(X\left(s_{1}+\cdots+s_{m+n}\right) \in U_{m+n}, \cdots, X\left(s_{1}+\cdots+s_{m}+s_{m+1}\right)\right. \\
\left.\quad \in U_{m+1} \mid X\left(s_{1}+\cdots+s_{m}\right) \in U_{m}, \cdots, X\left(s_{1}\right) \in U_{1}\right) \\
=p^{*} \psi(m+n) / p^{*} \psi(m) \\
=p^{*} E\left(U_{m+n}\right) T\left(s_{m+n}\right) \cdots E\left(U_{m+1}\right) T\left(s_{m+1}\right)\left[\frac{\psi(m)}{p^{*} \psi(m)}\right] .
\end{aligned}
$$

Using (4),

$$
\begin{aligned}
& \frac{\psi(m)(U, \xi)}{p^{*} \psi(m)}=E_{\left\{\psi(m) / p^{*} \psi(m)\right\}}\left[\xi I_{U}(X(0))\right] \\
& =\frac{E_{\{\psi(m)\}}\left[\xi I_{U}(X(0))\right]}{p^{*} \psi(m)} \\
& =\frac{E(\phi)\left[\xi_{s_{1}+\cdots+s_{m}}^{+} I_{U}\left(X\left(s_{1}+\cdots+s_{m}\right)\right), X\left(s_{1}\right) \in U_{1}, \cdots, X\left(s_{1}+\cdots+s_{m}\right) \in U_{m}\right]}{\phi\left(X\left(s_{1}\right) \in U_{1}, \cdots, X\left(s_{1}+\cdots+s_{m}\right) \in U_{m}\right)} \\
& =E(\phi)\left[\xi_{s_{1}+\cdots+s_{m}}^{+} I_{U}\left(X\left(s_{1}+\cdots+s_{m}\right)\right) \mid X\left(s_{1}\right) \in U_{1}, \cdots, X\left(s_{1}+\cdots+s_{m}\right) \in U_{m}\right] .
\end{aligned}
$$

Thus

$$
\begin{aligned}
& \phi\left(\Lambda_{s_{1}+\cdots+s_{m}}^{+} \mid X\left(s_{1}\right) \in U_{1}, \ldots, X\left(s_{1}+\cdots+s_{m}\right) \in U_{m}\right) \\
& =\phi\left[\Lambda_{s_{1}+\cdots+s_{m}}^{+} \mid E(\phi)\left(\xi_{s_{1}+\cdots+s_{m}}^{+} I_{U}\left(X\left(s_{1}+\cdots+s_{m}\right)\right) \mid\right.\right. \\
& \left.\left.X\left(s_{1}\right) \in U_{1}, \cdots, X\left(s_{1}+\cdots+s_{m}\right) \in U_{m}\right), \xi \in \Xi, U \in \Sigma\right]
\end{aligned}
$$

or

$$
\begin{aligned}
\phi\left(\Lambda_{t}^{+} \mid \mathcal{F}_{t}\right) & =\phi\left[\Lambda_{t}^{+} \mid E(\phi)\left(\xi_{t}^{+} I U(X(t)) \mid \mathcal{F}_{t}\right), \xi \in \Xi, U \in \Sigma\right] \\
& =\phi\left[\Lambda_{t}^{+} \mid I_{U}(X(t)) E(\phi)\left(\xi_{t}^{+} \mid \mathcal{F}_{t}\right), \xi \in \Xi, U \in \Sigma^{\prime}\right] \\
& =\phi\left[\Lambda_{t}^{+} \mid X(t), E(\phi)\left(\xi_{t}^{+} \mid \mathcal{F}_{t}\right), \xi \in \Xi\right]
\end{aligned}
$$

and so $X$ is a $\Xi$ process. 
On the other hand, if $X=\left(\hat{\Phi}, \hat{T}(t), \hat{E}(U), \hat{p}^{*}\right)$ is a $\exists$ process, then $(\hat{\Phi}, \hat{T}(t)$, $\left.\hat{E}(U), \hat{p}^{*}\right)$ is isomorphic to $\left(\Phi, T(t), E(U), p^{*}\right)$ via the mapping $\hat{\phi} \rightarrow \phi$ where

(1) $\Phi$ is the set of all functions $\phi$ mapping $\Sigma \times \Xi$ into $\mathcal{S}$ defined by $\phi(U, \xi)=E(\hat{\phi})\left(\xi I_{U}(X(0)), \hat{\phi} \in \dot{\Phi}\right.$.

(2) $T(t) \phi(U, \xi)=E(\hat{\phi})\left(\xi_{t}^{+} I_{U}(X(t))\right)$.

(3) $E(V) \phi(U, \xi)=\phi(U V, \xi)$.

(4) $p^{*} \phi=\phi(\delta, 1)$.

Clearly $T(t)$ is well defined on $\Phi$ since if $\hat{\phi}=\hat{\psi}$, then $\phi=\psi$ since $X$ is a $\Xi$ process. Also one clearly has

$$
\begin{aligned}
& p^{*} E\left(U_{n}\right) T\left(t_{n}\right) \cdots E\left(U_{1}\right) T\left(t_{1}\right) \phi \\
& \quad=\phi\left(X\left(t_{1}\right) \in U_{1}, \cdots, X\left(t_{1}+\cdots+t_{n}\right) \in U_{n}\right)=\hat{p}^{*} \hat{E}\left(U_{n}\right) \hat{T}\left(t_{n}\right) \cdots \hat{E}\left(U_{1}\right) \hat{T}\left(t_{1}\right) \hat{\phi}
\end{aligned}
$$

and so the isomorphism is proved.

6. Proof of Theorem V. We first note that $T$ satisfies condition 4 of Theorem IV since if $\xi=I_{V}(X(1))$, then

$$
\begin{aligned}
E(\phi)\left(\xi I_{U}(X(0))\right. & =E(\phi)\left(I_{V}(X(1)) I_{U}(X(0))=\phi(X(0) \in U, X(1) \in V)\right. \\
& =p^{*} E(V) T E(U) \phi=E(V) T E(U) \phi(S, \delta)=T E(U) \phi(V, \mathcal{S}) \\
& =-\int_{0}^{\infty} d v \int_{V} d u \int_{0}^{\infty} \frac{\partial}{\partial v}\left[B(x, u, u+v) E(U) \phi^{\prime}(d x, u+v)\right] \\
& =-\int_{0}^{\infty} d v \int_{V} d u \int_{U} \frac{\partial}{\partial v}\left[B(x, u, u+v) \phi^{\prime}(d x, u+v)\right] \\
& =\int_{V} d u \int_{U} \phi^{\prime}(d x, u)=\phi(U, V)=\phi(U, \xi) .
\end{aligned}
$$

Thus $X$ is a $\Xi_{0}$ process provided that $\Phi_{+}$is closed. We will show this later. Part (1) of the theorem is obvious. To prove part (2), we note that

$$
\begin{aligned}
& \operatorname{TE}\left(U_{n}\right) \cdots T E\left(U_{0}\right) \phi(U, V) \\
& =(-1)^{n} \int_{V} d v \int_{U} d u \int_{U_{n}} \cdots \int_{U_{0}} \frac{\partial}{\partial v} B\left(x_{n}, v, v+u\right) \\
& \quad \frac{\partial}{\partial v} B\left(x_{n-1}, x_{n}, x_{n}+u+v\right) \frac{\partial}{\partial v} B\left(x_{n-2}, x_{n-1}, x_{n-1}+x_{n}+u+v\right) \ldots \\
& \quad \frac{\partial}{\partial v} B\left(x_{0}, x_{1}, x_{1}+\cdots+x_{n}+u+v\right) \phi^{\prime}\left(d x_{0}, x_{1}+\cdots+x_{n}+u+v\right) d x_{1} \cdots d x_{n}
\end{aligned}
$$

and so a necessary and sufficient condition that $\phi \in \Phi_{+}$is that $p^{*} \phi=1$ and for all $U, U_{0}, \cdots, U_{n}$ 
$p^{*} E(U) T E\left(U_{n}\right) \cdots T E\left(U_{0}\right) \phi=T E\left(U_{n}\right) \cdots T E\left(U_{0}\right) \phi(U, S)$

$$
\begin{aligned}
& =(-1)^{n-1} \int_{U} d u \int_{U_{n}} \cdots \int_{U_{0}} \frac{\partial}{\partial u} B\left(x_{n-1}, x_{n^{\prime}} x_{n}+u\right) \frac{\partial}{\partial u} B\left(x_{n-2}, x_{n-1}+x_{n}+u\right) \\
& \cdots \frac{\partial}{\partial u} B\left(x_{0}, x_{1}, x_{1}+\cdots+x_{n}+u+v\right) \phi^{\prime}\left(d x_{0}, x_{1}+\cdots+x_{n}+u\right) d x_{1} \cdots d x_{n} \geq 0
\end{aligned}
$$

or equivalently that

$$
\begin{aligned}
& (-1)^{n-1} \int_{U_{0}} \frac{\partial}{\partial u} B\left(x_{n-1}, x_{n}, x_{n}+u\right) \frac{\partial}{\partial u} B\left(x_{n-2} x_{n-1} x_{n-1}+x_{n}+u\right) \\
& \cdots \frac{\partial}{\partial u} B\left(x_{0}, x_{1}, x_{1}+\cdots+x_{n}+u\right) \phi^{\prime}\left(d x_{0}, x_{1}+\cdots+x_{n}+u\right) \geq 0
\end{aligned}
$$

Letting $x_{2}=\cdots=x_{n}=u=0$ we see that a necessary condition that $\phi \in \Phi_{+}$is that for all nonnegative integers $n$,

$$
[-\partial / \partial x]^{n} \phi^{\prime}(U, x) \geq 0 .
$$

Thus $\phi^{\prime}(U, \cdot)$ is a completely monotone function on $R_{+}$. Conversely, if $\phi^{\prime}(U, \cdot)$ is a completely monotone function, then it is clearly in $\Phi_{+}$since the completely monotone functions are closed under products and the operators $-\partial / \partial x_{\text {. It follows, }}$ therefore, that $\Phi_{++}$consists of the functions

$$
\begin{aligned}
\phi_{a, b}(U, V) & =\int_{V} e^{-b v}, & & a \in U, \\
& =0, & & a \notin U,
\end{aligned}
$$

and so the proof of part (2) is complete. Part (3) follows, letting $a_{b}(a, y, U)=$ $a_{a, y}(U-b)$, from the fact that

$$
\begin{aligned}
\int_{0}^{\infty} e^{-\eta v} P(a, b ; U, d \eta) & =\int_{0}^{\infty} \int_{0}^{\infty} \phi_{\xi, \eta}(U, v) P(a, b ; d \xi, d \eta) \\
& =\left(T \phi_{a, b}\right)^{\prime}(U, v)=-\int_{U} \frac{\partial}{\partial v} B(a, u, u+v) e^{-b(u+v)} d y \\
& =-\int_{U} \frac{\partial}{\partial v} \int_{0}^{\infty} e^{-(b+\eta)(u+v)} a(a, u, d \eta) d u \\
& =\int_{U} \int_{0}^{\infty}(b+\eta) e^{-(b+\eta)(u+v)} a(a, u, d \eta) d u \\
& =\int_{U} \int_{b}^{\infty} \eta e^{-\eta u} e^{-\eta v} a_{b}(a, u, d \eta) d u \\
& =\int_{0}^{\infty} e^{-\eta v}\left[{ }^{-\infty, \infty)}(\eta) \int_{U} e^{-\eta u} a_{b}(a, u, d \eta) d u\right.
\end{aligned}
$$


which implies that

$$
\begin{aligned}
P(a, b ; U, V) & =\int_{V \cap[b, \infty)} \int_{U} e^{-\eta u} a_{b}(a, u, d \eta) d u \\
& =\int_{U} d u \int_{(V-b) \cap[0, \infty)} e^{-u(\eta+b)} a(a, u, d \eta)
\end{aligned}
$$

To prove part (4), we assume first that $\phi$ is a version of $X$. Then for all $0 \leq x \leq y$

$$
\begin{aligned}
\phi(X(n+1)< & \left.x \mid X(0)=x_{0}, \cdots, X(n)-x_{n^{\prime}} X(n+1)<y<X(n+1)+X(n+2)\right) \\
& -\frac{(-1)^{n} \int_{0}^{x} d x_{n+1} \int_{y-x_{n+1}}^{\infty} \frac{\partial}{\partial x_{n+2}} B\left(x_{n^{\prime}} x_{n+1}, x_{n+1}+x_{n+2}\right) \cdots \phi^{\prime}\left(d x_{0}, x_{1}+\cdots+x_{n+2}\right) d x_{n+2}}{(-1)^{n} \int_{0}^{y} d x_{n+1} \int_{y-x_{n+1}}^{\infty} \frac{\partial}{\partial x_{n+2}} B\left(x_{n^{\prime}} x_{n+1}, x_{n+1}+x_{n+2}\right) \cdots \phi^{\prime}\left(d x_{0}, x_{1}+\cdots+x_{n+2}\right) d x_{n}} \\
& =\frac{\int_{0}^{x} B\left(x_{n^{\prime}}, x_{n+1}, y\right) \frac{\partial}{\partial y} B\left(x_{n-1}, x_{n^{\prime}}, x_{n}+y\right) \cdots \phi^{\prime}\left(d x_{0}, x_{1}+\cdots+x_{n}+y\right) d x_{n+1}}{\int_{0}^{y} B\left(x_{n^{\prime}}, x_{n+1}, y\right) \frac{\partial}{\partial y} B\left(x_{n-1}, x_{n^{\prime}}, x_{n}+y\right) \cdots \phi^{\prime}\left(d x_{0}, x_{1}+\cdots+x_{n}+y\right) d x_{n+1}} \\
= & \int_{0}^{x} B\left(x_{n^{\prime}}, x_{n+1}, y\right) d x_{n+1} / \int_{0}^{y} B\left(x_{n^{\prime}}, x_{n+1}, y\right) d x_{n+1},
\end{aligned}
$$

On the other hand, suppose that

$$
\begin{aligned}
\phi\left(X(n+1)<x \mid \mathcal{F}_{n}, X(n+1)<y<X(n+1)+X(n+2)\right) \\
=\int_{0}^{x} B\left(x_{n}, \xi, y\right) d \xi / \int_{0}^{y} B\left(x_{n}, \xi, y\right) d \xi .
\end{aligned}
$$

Then

$$
\begin{aligned}
& \frac{\int_{0}^{x} B(X(n), \xi, y) d \xi}{\int_{0}^{y} B(X(n), \xi, y) d \xi}=\frac{\phi\left(X(n+1)<x<y<X(n+1)+X(n+2) \mid \mathcal{F}_{n}\right)}{\phi\left(X(n+1)<y<X(n+1)+X(n+2) \mid \mathcal{F}_{n}\right)} \\
& =\frac{E\left[\phi\left(X(n+1)<x<y<X(n+1)+X(n+2) \mid \mathcal{F}_{n+1}\right) \mid \mathcal{F}_{n}\right]}{E\left[\phi\left(X(n+1)<y<X(n+1)+X(n+2) \mid \mathcal{F}_{n+1}\right) \mid \mathcal{F}_{n}\right]} \\
& =\frac{\int_{0}^{x} \phi\left(X(n+2)>y-\xi \mid \mathcal{F}_{n}, X(n)=\xi\right) \phi\left(X(n+1)=d \xi \mid \mathfrak{F}_{n}\right)}{\int_{0}^{y} \phi\left(X(n+2)>y-\xi \mid \mathcal{F}_{n}, X(n+1)=\xi\right) \phi\left(X(n+1)=d \xi \mid \mathcal{F}_{n}\right)} \\
& =\frac{\int_{0}^{x} \phi\left(X(n+2)>y-\xi \mid \mathcal{F}_{n}, X(n+1)=\xi\right) \phi^{\prime}\left(X(n+1)=\xi \mid \mathcal{F}_{n}\right) d \xi}{\int_{0}^{y} \phi\left(X(n+2)>y-\xi \mid \mathfrak{F}_{n}, X(n+1)=\xi\right) \phi^{\prime}\left(X(n+1)=\xi \mid \mathcal{F}_{n}\right) d \xi} .
\end{aligned}
$$

Differentiating both sides of this eqaution with respect to $x$ and then letting $x=y$ yields 


$$
\begin{aligned}
\int_{0}^{y} \phi(X(n+2) & \left.>y-\xi \mid \mathcal{F}_{n}, X(n+1)=\xi\right) \phi^{\prime}\left(X(n+1)=\xi \mid \mathcal{F}_{n}\right) d \xi \\
& =\phi^{\prime}\left(X(n+1)=y \mid \mathcal{F}_{n}\right) \int_{0}^{y} B(X(n), \xi, y) d \xi .
\end{aligned}
$$

Thus

$$
\int_{0}^{y} B(X(n), \xi, y) d \xi=\frac{\int_{0}^{x} \phi\left(X(n+2)>y-\xi \mid F_{n}, X(n+1)=\xi \phi^{\prime}\left(X(n+1)=\xi \mid F_{n}\right) d \xi\right.}{\phi^{\prime}\left(X(n+1)=\left.y\right|^{\mathscr{F}}{ }_{n}\right)}
$$

Differentiating with respect to $x$ and then replacing $y-x$ by $x_{n+2}$ and $x$ by $x_{n+1}$ yièlds the equation

$$
\begin{aligned}
& \phi\left(X(n+2)>x_{n+2} \mid \mathcal{F}_{n}, X(n+1)=x_{n+1}\right) \\
& =B\left(X(n), x_{n+1}, x_{n+1}+x_{n+2}\right) \frac{\phi^{\prime}\left(X(n+1)=x_{n+1}+x_{n+2} \mid \mathcal{F}_{n}\right)}{\phi^{\prime}\left(X(n+1)=x_{n+1} \mid \mathcal{F}_{n}\right)}
\end{aligned}
$$

or

$$
\begin{aligned}
\phi^{\prime}(X(n+2) & \left.=x_{n+2} \mid X(0)=x_{0}, \cdots, X(n+1)=x_{n+1}\right) \\
& =-\frac{\partial}{\partial x_{n+2}} B\left(x_{n}, x_{n+1}, x_{n+1}+x_{n+2}\right) \frac{\phi^{\prime}\left(X(n+1)=x_{n+1}+x_{n+2} \mid X(0)=x_{0}, \ldots, X(n)=x_{n}\right)}{\phi^{\prime}\left(X(n+1)=x_{n+1} \mid X(0)=x_{0}, \cdots, X(n)=x_{n}\right)} .
\end{aligned}
$$

It therefore follows by induction on $n$ that

$$
\begin{aligned}
\phi^{\prime}(X(0)= & \left.x_{0}, \cdots, X(n)=x_{n}\right) \\
= & \phi^{\prime}\left(X(0)=x_{0}\right) \phi^{\prime}\left(X(1)=x_{1} \mid X(0)=x_{0}\right) \cdots \phi^{\prime}\left(X(n)=x_{n} \mid X(0)=x_{0}, \cdots, X(n-1)=x_{n-1}\right) \\
= & -\phi^{\prime}\left(X(0)=x_{0}\right) \phi^{\prime}\left(X(1)=x_{1} \mid X(0)=x_{0}\right) \cdots \phi^{\prime}\left(X(n-2)=x_{n-2} \mid X(0)=x_{0}, \ldots, X(n-3)=x_{n-3}\right) \\
& \cdot \frac{\partial}{\partial x_{n}} B\left(X(n-2), x_{n-1}, x_{n-1}+x_{n}\right) \phi^{\prime}\left(X(n-1)=x_{n-1}+x_{n} \mid X(0)=x_{0}, \cdots, X(n-2)=x_{n-2}\right) \\
= & -\frac{\partial}{\partial x_{n}} B\left(x_{n-2}, x_{n-1}, x_{n-1}+x_{n}\right) \phi^{\prime}\left(X(0)=x_{0}, \cdots, X(n-2)=x_{n-2}, X(n-1)=x_{n-1}+x_{n}\right) \\
= & (-1)^{n} \frac{\partial}{\partial x_{n}} B\left(x_{n-2}, x_{n-1}, x_{n-1}+x_{n}\right) \frac{\partial}{\partial x_{n}} B\left(x_{n-3^{\prime}} x_{n-2}, x_{n-2}+x_{n-1}+x_{n}\right) \\
& \cdots \frac{\partial}{\partial x_{n}} B\left(x_{0}, x_{1}, x_{1}+\cdots+x_{n}\right) \phi^{\prime}\left(X(0)=x_{0}, X(1)=x_{1}+\cdots+x_{n}\right)
\end{aligned}
$$

and so, comparing with equation (1) we see that $\phi$ is a $\Xi_{0}$ process. Finally, we note that $\Phi$ is weak* closed since $\Phi_{++}$is weak* closed. That is to say, if $\phi_{a_{n}, b_{n}}$ converges weak* to $\phi$, then for any continuous function $f$ mapping $R_{+} \times$ $R_{+} \rightarrow R$ one has 


$$
\begin{aligned}
\int_{0}^{\infty} \int_{0}^{\infty} f(u, v) p^{*} E(d v) T E(d u)\left[\phi_{a_{m}, b}-\phi_{a_{n}, b}\right] \\
=\int_{0}^{\infty} \int_{0}^{\infty} f(u, v) \phi_{a_{m}, b}(d u, d v)-\int_{0}^{\infty} \int_{0}^{\infty} f(u, v) \phi_{a_{n}, b_{n}}(d u, d v) \\
=\int_{0}^{\infty} f\left(a_{m}, v\right) e^{-b_{m} v} d v-\int_{0}^{\infty} f\left(a_{n}, v\right) e^{-b_{n} v} d v \rightarrow 0 .
\end{aligned}
$$

Clearly this implies the existence of $a$ and $b$ such that $a_{n} \rightarrow a$ and $b_{n} \rightarrow b$ and $\phi_{a_{n}, b_{n}} \rightarrow \phi_{a_{0} b}=\phi$.

7. Example where $\bar{X}^{*} \neq X^{*}$. Consider the discrete time temporally homogeneous Markov process $Y$ on $\{1,2,3\}$ whose transition probability matrix is

$$
\left[\begin{array}{ccc}
0 & 1 & 0 \\
0 & 1 / 2 & 1 / 2 \\
1 & 0 & 0
\end{array}\right]
$$

and whose initial distribution is $(0,0,1)$. Let $\theta$ be the mapping of $\{1,2,3\}$ onto $\{1,2\}$ defined by $f(1)=f(2)=1$ and $f(3)=2$, and let $\mu$ be the probability measure generated by $\theta Y$ on $(\Omega, \mathcal{F})$ where $\Omega$ is the set of all functions mapping the nonnegative integers into $\{1,2\}$. The reader can easily check that $X(\mu)=$ $\left(\Phi, T, E(U), p^{*}, \phi_{0}\right)$ where

$$
\begin{gathered}
\Phi=R^{3}, \\
T=\left[\begin{array}{lll}
0 & 1 & 0 \\
0 & 1 / 2 & 1 / 2 \\
1 & 0 & 0
\end{array}\right], \\
E(\{1\})=\left[\begin{array}{lll}
1 & 0 & 0 \\
0 & 1 & 0 \\
0 & 0 & 0
\end{array}\right], \quad E(\{2\})=\left[\begin{array}{lll}
0 & 0 & 0 \\
0 & 0 & 0 \\
0 & 0 & 1
\end{array}\right] . \\
p^{*}\left(x_{1}, x_{2}, x_{3}\right)=x_{1}+x_{2}+x_{3}, \\
\phi_{0}=(0,0,1) .
\end{gathered}
$$

Furthermore,

$$
\Phi_{++}=\{(1,0,0),(0,1,0),(0,0,1)\}
$$


and $X\left(\phi_{0}\right)^{*}$ is the temporally homogeneous Markor process on $\Phi_{++}$with transition probability matrix

$$
\left[\begin{array}{ccc}
0 & 1 & 0 \\
0 & 1 / 2 & 1 / 2 \\
1 & 0 & 0
\end{array}\right] .
$$

Thus identifying 1 with $(1,0,0), 2$ with $(0,1,0)$ and 3 with $(0,0,1)$ we see that $X(\phi)^{*}=Y$. On the other hand, $\bar{X}^{*}$ lives on $\bar{\Phi}_{++}=\{(1,0,0),(-1,2,0)$, $(0,0,1)\}$ and has the transition probability matrix

$$
\left[\begin{array}{ccc}
1 / 2 & 1 / 2 & 0 \\
0 & 0 & 1 \\
1 & 0 & 0
\end{array}\right] .
$$

\section{REFERENCES}

1. Robert R. Bush and Frederick Mosteller, Stochastic models for learning, Wiley, New York; Chapman \& Hall, London, 1955. MR 16, 1136.

2. N. Dunford and J. T. Schwartz, Linear operators. I: General theory, Pure and Appl. Math., vol. 7, Interscience, New York, 1958. MR 22 \#8302.

3. Dudley Paul Johnson, On a class of stochastic processes and its relationship to infinite particle gases, Trans. Amer. Math. Soc. 132 (1968), 275-295. MR 41 \#1108.

4. - On a class of stochastic processes with two states and continuous time parameter, Trans. Amer. Math. Soc. 142 (1969), 257-267. MR 39 \#7687.

5. Dudley Paul Johnson, Markov process representations of general stochastic processes, Proc. Amer. Math. Soc. 24 (1970), 735-738. MR 41 \#6303.

6. - Hitting time distribution for general stochastic processes, Ann. Math. Statist. 43 (1972), 1716-1718.

7. P. A. Meyer, Probability and potentials, Blaisdell, Waltham, Mass., 1966. MR $34 \# 5119$.

DEPARTMENT OF MATHEMATICS, UNIVERSITY OF CALGARY, CALGARY, ALBERTA, CANADA 